\title{
THIF-8
}

\section{Optimization of 3D Multilayer RF Components Using the Design of Experiments (DOE) Technique}

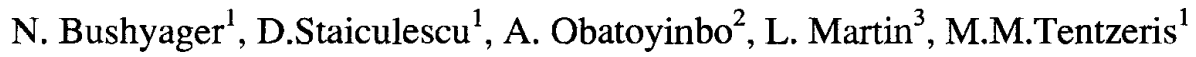 \\ ${ }^{1}$ Georgia Electronic Design Center, School of ECE, Georgia Institute of Technology, \\ Atlanta, GA, 30332-0269, U.S.A. \\ Fax: (404) 894-0222, Email: daniela@ece.gatech.edu \\ ${ }^{2}$ HRL Laboratories LLC, Malibu, CA, 90265, U.S.A. \\ ${ }^{3}$ Motorola, 8000 Sunrise Blvd., Plantation, FL, 33322, USA
}

\begin{abstract}
The successful use of the Design of Experiments (DOE) approach in an optimization feasibility study for two microwave balanced to unbalanced transformers (baluns) is presented. The medium of interest is the multi-layer Low Temperature Cofired Ceramic (LTCC) and two different topologies, one microstrip and one stripline, are investigated. The design goals are perfectly balanced output from $2-3 \mathrm{GHz}$ and a resonant frequency of exactly $2.4 \mathrm{GHz}$. It is demonstrated, using only eight simulations, that perfectly balanced outputs are not possible under the given conditions in the case of the microstrip balun. Nevertheless, the stripline balun can be optimized due to its almost symmetrical structure, and both simulations and measurement results verify the conclusions. The DOE method is very simple to implement and gives a clear understanding of the system behavior at the beginning of the design process, reducing the amount of work required for achieving the design goals by orders of magnitude compared to the widely used trial-anderror approach.
\end{abstract}

Index Terms - Multilayer modules, balun, optimization, design of experiments.

\section{INTRODUCTION}

The increased demands for compactness and functionality in modern $3 \mathrm{D}$ modules and packages [1] make the design and optimization processes of such systems more and more challenging. Existing optimization packages included in the commercial electromagnetic simulators like High Frequency Structure Simulator (HFSS), often do not take into account the specific effect of each of the factors involved in the design process and the degree of interaction between them, thus leading to time-consuming "trial-and-error" approaches. Especially for the complicated 3D architectures of $\mathrm{RF} / \mathrm{microwave}$ multilayer modules, a comprehensive and sophisticated tool is necessary to account for all the complex phenomena such as coupling and fringing effects. Alternative optimization methods suited to this kind of complexity are Neural Networks [2] and Genetic Algorithms [3]. These are very precise methods which unfortunately require a hefty amount of prior knowledge and are computationally complex and time consuming. Design of Experiments (DOE) overcomes all these disadvantages. It provides a thorough understanding of all the factors involved in the design process, and identifies which ones are more significant, which ones are not significant at all, how they interact with each other, if the goals are achievable in the given conditions, etc. Most importantly, the method is very easy to implement with a negligible computational overhead. This paper presents the successful use of DOE in the design and optimization of two $2.4 \mathrm{GHz}$ multilayer baluns in microstrip and stripline configurations.

\section{BALUN BACKGROUND}

A balun basically transforms a single-ended network to a differential one and vice-versa. Active [4] and MMIC [5] baluns have been reported in the literature, but they are plagued either by the high DC power consumption and noise figure or by the increased size on chip which increases the cost of the final product substantially. The drive in recent years towards System On a Package (SOP) and module development requires the moving of certain components off-chip.

For our study, the basic coupled line balun concept presented in Fig. 1 has been used [5].

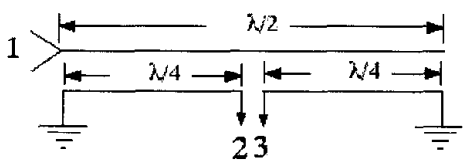

Fig. 1. Conceptual representation of the coupled line balun.

Two $\lambda / 4$ shorted lines are placed next to a $\lambda / 2$ open line such that they couple energy from the open line. A signal incident at port 1 induces signals at port 2 and port 3 with equal amplitudes and opposite phases. 
For this study, 20-layer LTCC substrate is used, with the following characteristics: $\varepsilon_{\mathrm{r}}=7.8, \tan \delta=0.005$, layer thickness $=3.7 \mathrm{mil}$. In order to save space, the lines have been implemented as spirals, both in stripline and microstrip topologies. Fig. 2 and 3 present exploded views of the microstrip and stripline baluns respectively. The optimization of these structures is quite challenging since the close proximity of the turns introduces parasitics and sometimes destroys the symmetry of the topology. On the other hand, the conventional optimization would require the variation of a large number of geometrical parameters and a very large number of simulations.

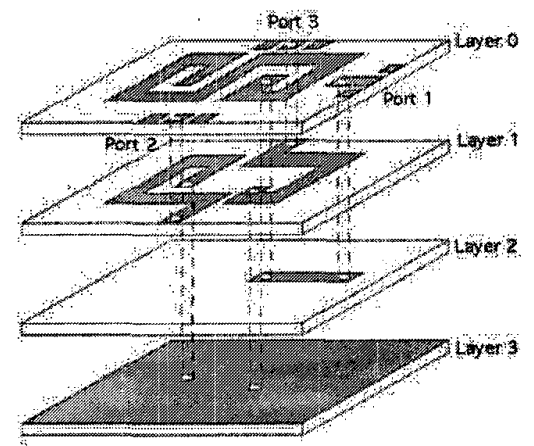

Fig. 2. Microstrip implementation of multilayer balun.

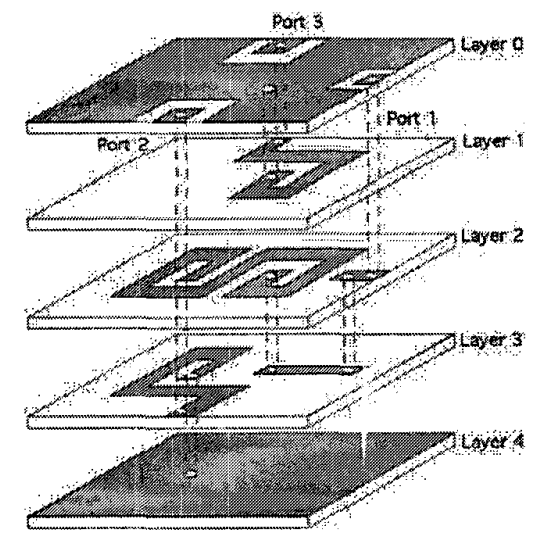

Fig. 3. Stripline implementation of multilayer balun

In this paper, the Design of Experiments (DOE) tool will be applied at the beginning of the design process for a thorough understanding on how various design parameters can be changed to achieve optimal performance. The design goals are a resonant frequency of $2.4 \mathrm{GHz}$, as well as excellent amplitude balance and a consistent phase imbalance at the output from $2-3 \mathrm{GHz}$. It is expected that the optimization of the stripline topology will be easier due to its symmetry. Also, since the outputs are on different layers, one can expect good isolation characteristics between ports 2 and 3 . However, with the microstrip topology, the structure is not symmetric and the port 3 line is somewhat shortened compared to the port 2 line. This is due to the fact that both outputs are taken from the same level and allowance has to be given for the via that connects the portion of the open line on layer 2 to the rest of the line on layer 1 .

\section{DOE BACKGROUND}

A design of experiments is a series of tests in which a set of input variables or factors is purposely changed so that the experimenter can observe and identify the reasons for changes in the output response. Previous work shows the use of design of experiments in modeling of RF/microwave circuits [6]. The factorial designs are used in experiments involving several factors where the goal is the study of the joint effects of the factors on a response. Prior knowledge of the analyzed system is required for choosing the factors and their studied ranges. The $2^{k}$ factorial design is the simplest one, with $k$ factors at 2 levels each. It provides the smallest number of runs for studying $k$ factors and is widely used in factor screening experiments [7].

This paper shows the first use of the DOE in a feasibility study of the optimization of a circuit. In other words, for the two balun topologies presented in the previous section, will be demonstrated that, under the given design conditions, the microstrip balun cannot completely satisfy the balance design requirements for any frequency between $2-3 \mathrm{GHz}$ that is different than the design frequency. This is a very important conclusion, in any case a designer tries to achieve the unachievable. When confronted with such challenges, the designers often spend a lot of time trying to get results rather than just do a systematic factor screening at the beginning of the design process.

First, electromagnetic analysis of the system helps identify the important factors to be considered. Then these factors are included in a factorial experiment and the outputs are obtained and recorded using a full-wave timedomain simulator. The optimization constraints are afterwards applied and the values of the input variables that best satisfy all of them are calculated.

\section{MICROSTRIP BALUN OPTTMIZATTON}

The first case to be considered is the microstrip topology, which is of great interest due to its wide use in RF and microwave circuits. The preliminary simulation of the structure in Fig. 2 shows poor amplitude balance between ports 2 and 3, as shown in Fig. 4. A detailed look at the field distribution shows that there is a lot of 
coupling at the center of the structure. This is due to the fact that the vertical center part of the open line on Layer 0 does not couple with the short lines on Layer 1 and causes coupling with the neighboring lines on the open spiral line. Also, strong coupling is present between the corners of the spiral open on Layer 0 and the two lines connecting the shorts to the ports 2 and 3 on Layer 1 . All these coupling effects are illustrated in Fig. 5 on a top view of the structure.

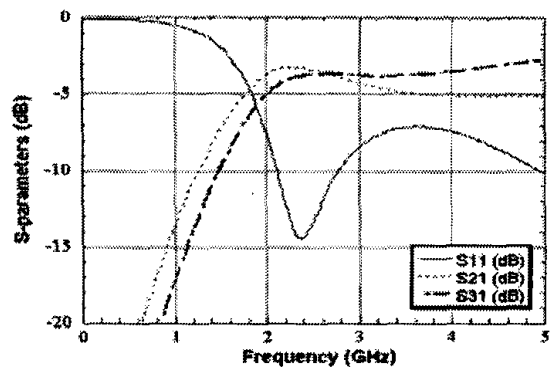

Fig. 4. S-parameters of initial microstrip balun

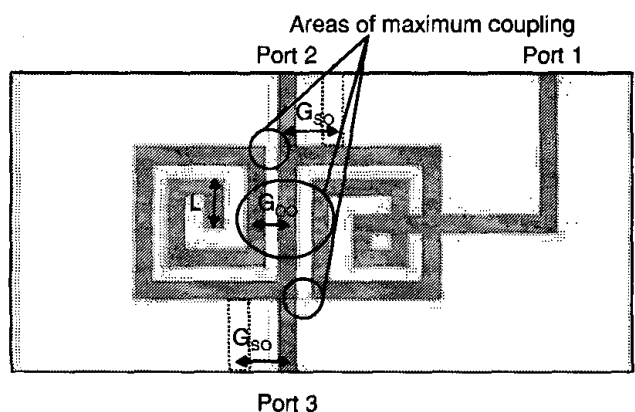

Fig. 5. Top view emphasizing the coupling effects of microstrip balun.

Therefore, two factors have already been identified for the experiment: $G_{o o}$, or the open-to-open gap, and $G_{\text {so }}$, or the distance the output lines for ports 2 and 3 are moved from the initial position for coupling reduction, as shown in Fig. 5. A third factor L, representing the length by which the lines are shrunk at one end, is added to the experiment for compensating the imbalance between $S_{21}$ and $S_{31}$. A full factorial experiment with three factors consists of $2^{3}=8$ treatment combinations. The two levels chosen for each input variable have been controlled by the fabrication process and are presented in Table 1.

Table 1. Variables for the $2^{3}$ experiment

\begin{tabular}{|c|c|c|c|}
\hline Variable & $\mathbf{L}$ (mil) & $\mathbf{G}_{\text {so }}$ (mil) & $\mathbf{G}_{\mathbf{0 0}}(\mathbf{m i l})$ \\
\hline "G_"level & 0 & 0 & 6 \\
\hline "ct" level & 30 & 13 & 19 \\
\hline
\end{tabular}

The output variables chosen are $\Delta_{2 \mathrm{GHz}}$ and $\Delta_{3 \mathrm{GH} z}$, which are the differences between $S_{21}$ and $S_{31}$ at $2 \mathrm{GHz}$ and $3 \mathrm{GHz}$ respectively, as well as the resonant frequency $f_{\text {res. }}$. The optimization goal is: $\Delta_{2 \mathrm{GHz}}=0 ; \Delta_{3 \mathrm{GHz}}=0 ; \mathrm{f}_{\mathrm{res}}=2.4 \mathrm{GHz}$.

The eight simulations have been run in MicroStripes TLM Modeler and the results are presented in Table 2.

Table 2. $2^{3}$ experiment

\begin{tabular}{|c|c|c|c|c|c|c|}
\hline $\begin{array}{c}\mathbf{R u} \\
\mathbf{n}\end{array}$ & $\mathbf{L}$ & $\mathbf{G}_{\text {so }}$ & $\mathbf{G}_{\mathbf{0 0}}$ & $\begin{array}{c}\mathbf{f}_{\text {res }} \\
(\mathbf{G H z})\end{array}$ & $\begin{array}{c}\Delta_{\mathbf{2} \mathbf{G H z}} \\
(\mathbf{d B})\end{array}$ & $\begin{array}{c}\mathbf{\Delta}_{\mathbf{3 G H z}} \\
(\mathbf{d B})\end{array}$ \\
\hline $\mathbf{1}$ & - & - & - & 2.37 & 1.4 & 0.7 \\
\hline $\mathbf{2}$ & + & - & - & 2.42 & 1.1 & 0.8 \\
\hline $\mathbf{3}$ & - & + & - & 2.37 & 1.2 & 0.5 \\
\hline $\mathbf{4}$ & + & + & - & 2.42 & 0.9 & 0.9 \\
\hline $\mathbf{5}$ & - & - & + & 2.29 & 1.6 & 0.6 \\
\hline $\mathbf{6}$ & + & - & + & 2.40 & 1.0 & 0.9 \\
\hline $\mathbf{7}$ & - & + & + & 2.30 & 0.9 & 0.7 \\
\hline $\mathbf{8}$ & + & + & + & 2.41 & 0.8 & 0.9 \\
\hline
\end{tabular}

Analysis of Variance (ANOVA) statistical analysis [7] has been performed using a user-friendly specialized commercial software. ANOVA reveals the statistical significance of all the input variables and of their interactions and generates regression models of the outputs as a function of the inputs. In this case:

$\Delta_{2 \mathrm{GHz}}=1.51-0.010833 \mathrm{~L}-0.025 \mathrm{G}_{\mathrm{so}}-0.005769 \mathrm{G}_{\mathrm{oo}}$

$\Delta_{3 \mathrm{GHz}}=0.577+0.00833 \mathrm{~L}+0.0038462 \mathrm{G}_{\mathrm{oO}}$

The most significant factor is found to be L. By fixing the other two variables at the most convenient level for achieving the optimal, the two values needed for $\mathbf{L}$ to satisfy both $\Delta_{2 \mathrm{GHz}}=0$ and $\Delta_{3 \mathrm{GHz}}=0$ are $\mathrm{L}_{1}=106$ mils and $\mathbf{L}_{2}=-72$ mils. These two conditions cannot be satisfied at the same time, and this renders the optimization of the microstrip balun impossible under the described conditions. The two transmission coefficients for ports 2 and 3 cannot satisfy the imbalance requirements in the studied bandwidth $(2-3 \mathrm{GHz})$ because the two lines have to be shrunk and elongated at the same time. Obviously, a trial-and-error approach where the designer changes the factors in a random manner could not have given the same response after only eight simulations. Genetic algorithms often require hundreds of simulations for optimization, while the trial-and-error approach can not give an understanding of the system after a very large number of simulations, especially when interactions are involved.

\section{STRIPLINE BALUN OPTTMTZATTON}

In the stripline case, the optimization includes only two factors: $G_{o o}$, or the open-to-open gap, and $G_{s o}$, or the short-to-open gap, since there is no need to compensate for any asymmetry in the geometry. A simple $2^{2}$ 
experiment is run for the same values of the two variables as the ones shown in Table 1. The optimal is achieved for the minimum values, $\mathrm{G}_{\mathrm{so}}=0$ and $\mathrm{G}_{\mathrm{oo}}=6$. The simulation results of the optimized structure are shown in Fig. 6.

The optimized balun has been fabricated and the measurements show very good agreement with the simulations for both the amplitude and phase imbalance. The results are presented in Fig. 7 and Fig. 8.

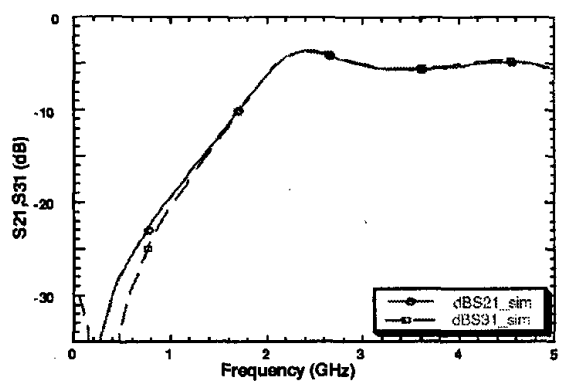

Fig. 6. S21 and S31 simulation for stripline balun.

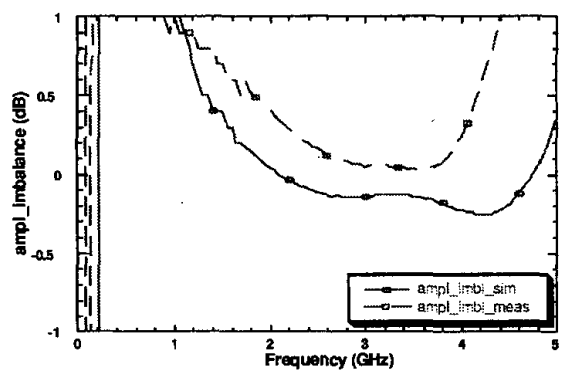

Fig. 7. Amplitude imbalance measurement vs. simulation for stripline balun.

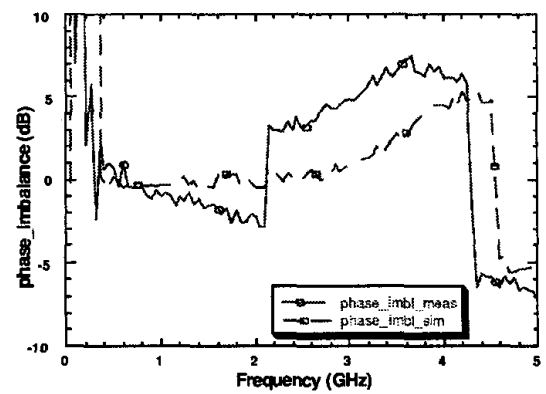

Fig. 8. Phase imbalance measurement vs. simulation for stripline balun.

\section{CONCLUSION}

The Design of Experiments approach has been combined with full wave simulations first for studying the feasibility of the optimization of two multilayer baluns and then for optimizing the feasible one. The two spiral baluns have been designed in microstrip and stripline topologies. The experiment was very simple to implement and provided a thorough understanding of the issues to be confronted to in the optimization process. For the microstrip topology, it was found that it was not possible to obtain a satisfactory amplitude balance for the entire frequency band. This was due to the asymmetries associated with the microstrip design. For the stripline topology, optimization was achieved and test structures were fabricated to validate the simulations. The measurements showed good agreement with the simulated structures for both the amplitude and the phase imbalance. It has to be noted that in both cases the amount of required simulations was a very small fraction (more than an order of magnitude) with respect to any other optimization approaches.

By extending this approach to carefully investigate the behavior of a complex system such as a 3D multilayer module at the beginning of the design process, the designer can save a lot of time, shorten the design cycle of added functions and achieve the design goals in a simple and elegant manner.

\section{ACKNOWLEDGEMENT}

The authors wish to acknowledge the support of the NSF CAREER ECS-9984761, the NSF ECS-0313951, and the GT-Packaging Research Center.

\section{REFERENCES}

[1] J. Laskar, A. Sutono, C.-H. Lee, M. Davis, M. Maeng, N. Lal, K. Lim, S. Pinel; M. Tentzeris, A. Obatoyinbo, "Development of integrated 3D radio front-end system-onpackage (SOP)" - 2003 GaAs IC Symp. Dig., pp. $215-218$, Oct. 2001.

[2] Hecht-Nielsen, R.: "Neurocomputing", New York, Addison-Wesley, 1990.

[3] N. Chaiyaratana, A.M.S. Zalzala: "Recent developments in evolutionary and genetic algorithms: theory and applications", Second International Conference On Genetic Algorithms In Engineering Systems, Innovations And Applications, pp. $270-277$, Sept. 1997.

[4] K.W. Kobayashi, "A Novel HBT Active Transformer Balanced Schottky Diode Mixer," Proc. IEEE MTT-S Int. Microwave Symp., vol.3, pp. 947-950, June 1996.

[5] M.C. Tsai, "A New Compact Wideband Balun," IEEE Microwave-Millimeter Wave Monolithic Circuits Symp. Dig., pp. 123-125, June 1993.

[6] D.Staiculescu, J.Laskar, E.M.Tentzeris, "Design Rule Development for Microwave Flip-Chip Applications", IEEE Trans. Microwave Theory \& Tech., Vol.48, No.9, pp.1476-1481, September 2000.

[7] D.C. Montgomery, Design and Analysis of Experiments, New York: J. Wiley \& Sons, 1997. 